Reportes de caso / Case reports

\title{
Granuloma telangiectásico bucal: lesión sangrante tratada con láser de diodo de 940nm en paciente pediátrico. Reporte de caso
}

\author{
Oral telangiectatic granuloma: bleeding lesion treated with a 940nm diode laser \\ in a pediatric patient. Case report
}

\author{
María Burgos Anaya1 (D), Zuly Garaviz Álvarez (D), Camila Giraldo Quintana (D), José Llamas García² (D), \\ David Rico Velasco $^{3}$ (D) Jonathan Harris Ricardo ${ }^{4}$ (D)
}

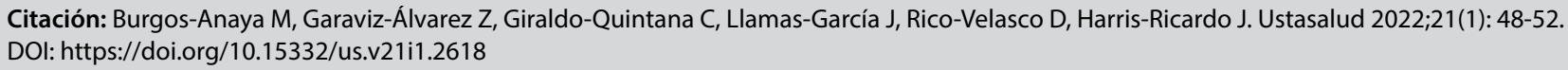

Licencia Creative Commons

\section{(c) (1) () $\Theta$} lo tanto, los lectores pueden acceder libremente a los artículos en su formato .pdf, igualmente podrán descargarlos y difundirlos; sin embargo no podrán modificarlos o alterarlos, adicionalmente se debe reconocer la autoría de las personas que figuran en las publicaciones, pero estas no podrán ser comercializadas.

\begin{abstract}
1 Estudiante de Odontología, Corporación Universitaria Rafael Núñez. Colombia.

2 Odontólogo, Mastership Láser Odontología, Especialista en Odontopediatría y Ortopedia Maxilar, Corporación Universitaria Rafael Núñez. Colombia.

3 Odontólogo, Especialista en Periodoncia, Corporación Universitaria Rafael Núñez. Colombia.

4 Odontólogo. Magíster en Microbiología Molecular, Especialista en Estomatología y Cirugía Oral. Corporación Universitaria Rafael Núñez Cartagena, Colombia.
\end{abstract}

Autor de correspondencia: Jonathan Harris Ricardo.

Correo electrónico:

Jonathan.harris@curnvirtual.edu.co

\section{Resumen}

El granuloma telangiectásico bucal es una lesión hiperplásica benigna que ocurre en respuesta a un traumatismo o irritación crónica en la cavidad oral. El aspecto histológico característico comprende un tejido de granulación típico con una proliferación de pequeños vasos sanguíneos de paredes delgadas en el tejido conectivo laxo y el tratamiento de elección es el quirúrgico. El propósito del trabajo es reportar el caso de un paciente pediátrico con granuloma telangiectásico bucal sangrante, en el que se describen las características clínicas, histológicas y la terapéutica con láser de diodo con buenos resultados posquirúrgicos.

Palabras clave: Granuloma piogénico, placa dental, hemorragia, coagulación por láser.

\section{Abstract}

Oral telangiectatic granuloma is a benign hyperplastic lesion that occurs in response to trauma or chronic irritation in the oral cavity. The characteristic histological appearance comprises a typical granulation tissue with a proliferation of small, thin-walled blood vessels in the loose connective tissue and the treatment of choice is surgical. The purpose of the work is to report the case of a pediatric patient with bleeding oral telangiectatic granuloma, in which the clinical and histological characteristics and diode laser therapy with good postoperative results are described.

Keywords: Pyogenic granuloma, dental plaque, hemorrhage, laser coagulation.
Recibido para publicación: 13 de septiembre de 2021 Aceptado para publicación: 6 de octubre de 2021 


\section{INTRODUCCIÓN}

El granuloma telangiectásico bucal (GTB), anteriormente llamado granuloma piógeno, es una neoplasia benigna epitelial con compromiso vascular, que suele presentarse como una lesión de fácil sangrado, pueden ocurrir en cualquier grupo de edad, con una incidencia mayor en la segunda y quinta década de vida, las mujeres suelen verse más afectadas que los hombres [1]. Con respecto a la edad, Asnaashari y otros en el 2015 afirmaron que también se manifiesta en edades tempranas y reportaron un caso de un niño de seis años [2].

La etiología se ha relacionado con aspectos como la presencia de espículas óseas, uso de aparatos de ortodoncia, fragmentos radiculares, irritación gingival, placa, cálculos y traumatismos [3]. Clínicamente el crecimiento es rápido, puede llegar hasta $2 \mathrm{~cm}$ en varias semanas, la lesión varía en colores de rosado pálido a rojo intenso, dependiendo de la cantidad de vasos sanguíneos y grado de inflamación, es de superficie lobulada, consistencia blanda, base sésil o pediculada y sangrado espontáneo, se caracteriza por mostrarse en distintos lugares como en la encía, labio, lengua, mucosa oral, paladar duro y piso de boca [4]. Patrón y otros, reportaron dos casos de pacientes con GTB y describieron que una de las características comunes en ambos casos fue la tendencia al sangrado de las lesiones con el mínimo rose, generando hemorragias continuas de leves a moderadas [5].

Su aspecto histológico comprende tejido de granulación típico, con infiltrados de células inflamatorias y una proliferación subepitelial característica de pequeños vasos sanguíneos de paredes delgadas en el tejido conectivo laxo [6].

Tradicionalmente el tratamiento de elección es la extirpación quirúrgica con bisturí, también otros métodos como escleroterapia, cauterización y el uso de láser [7]. La tecnología láser se está utilizando ampliamente en odontología, Espitia y otros en el 2018 afirmaron que el láser de diodo es una excelente opción en el tratamiento del GTB, por ser mínimamente invasivos, con mejor precisión de corte, bajo riesgo de infecciones posoperatorias, mejor hemostasia, minimiza el dolor, proporciona buena respuesta biológica, fácil aplicación y genera una mejor aceptación en los pacientes frente a las técnicas quirúrgicas tradicionales [8].
El propósito del presente trabajo es describir los resultados clínicos de la terapéutica con láser de diodo de $940 \mathrm{~nm}$, en un caso de granuloma telangiectásico con abundantes vasos capilares y tendencia al sangrado excesivo de un paciente pediátrico.

\section{PRESENTACIÓN DEL CASO}

Paciente masculino de 11 años, remitido al servicio de medicina oral de la Clínica Odontológica de la Corporación Universitaria Rafael Núñez, por presentar lesión de gran tamaño en labio inferior, afectando la estética y generando molestia al hablar y comer. En la anamnesis el paciente reportó presentar la lesión desde hace 6 meses aproximadamente, con historia previa de traumática (mordedura), que aumentó progresivamente de tamaño con el pasar del tiempo, asintomática y con sangrado espontáneo constante. Los antecedentes médicos personales y familiares no eran relevantes para el caso, durante la exploración de órganos y sistemas no presentó alteraciones considerables.

En el examen clínico se evidenció lesión tumoral en semimucosa labial inferior, del lado izquierdo, de aproximadamente $2 \mathrm{~cm}$ de diámetro, superficie irregular con zonas blancas y otras rojas, base pediculada, consistencia blanda a la palpación y sangrado activo (Figuras 1A, 1B).
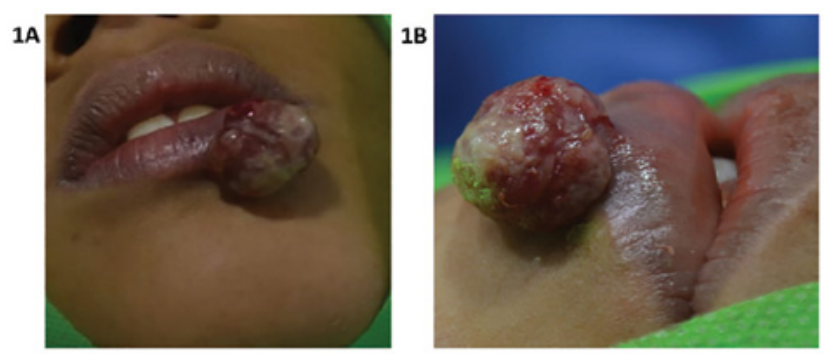

Figura 1A y 1B. Lesión tumoral en semimucosa labial inferior. Fuente: Autores.

Se estableció impresión clínica con granuloma telangiectásico bucal, teniendo en cuenta lo expresado por el paciente en la anamnesis, antecedentes de la lesión y el examen intraoral. Se llevó a cabo una junta odontológica con especialistas en cirugía oral, estomatología, periodoncia y odontopediatría, en la que se decidió hacer procedimiento quirúrgico aplicando láser de diodo de $940 \mathrm{~nm}$, se le explicó al niño y a los padres la técnica quirúrgica, posibles complicaciones, 
ventajas de la terapéutica y divulgación del caso en revista científica, los cuales estuvieron de acuerdo y se firmó el consentimiento informado por parte del acudiente. Antes de efectuar el procedimiento quirúrgico, se ordenaron exámenes paraclínicos como hemograma, recuento plaquetario, tiempo de protrombina, tiempo parcial de tromboplastina y glucemia, los cuales reportaron valores normales.

La cirugía se realizó bajo anestesia local, se aplicó técnica infiltrativa submucosa, con Roxicaína $2 \%$ sin epinefrina. Con respecto a la técnica, el protocolo para la aplicación del láser de diodo fue de $940 \mathrm{~nm}$ de longitud de onda, punta E3-4, modo continuo, potencia de 0,5 a 1,5 watts durante 10 segundos sobre la base de la lesión, realizando un rote de forma elíptica, con poco sangrado (Figura 2). La herida se suturó con hilo absorbible, se hizo un punto interno para generar hemostasia y puntos externos para confrontar los bordes de la herida. Se prescribió analgésico terapia y recomendaciones postoperatorias. El espécimen que se extrajo tenía un tamaño de $2 \mathrm{~cm}$ de diámetro (Figura 3 ), el cual se sumergió en un frasco con formol al 10\% y se envió para estudio histopatológico.

La descripción microscópica del reporte histopatológico describe epitelio escamoso hiperqueratósico, sin cambios displásicos, infiltrado inflamatorio con predominio de neutrófilos, abundantes vasos capilares y negativo para malignidad, compatible con granuloma telangiectásico (Figura 4).

Se hizo control clínico posquirúrgico a los 8 días, en el que se observó cicatrización del tejido, con zonas rojas inflamatorias y los nudos del hilo de sutura sueltos (Figura 5A). Se realizó un nuevo control clínico a los 15 días, en el que se observó disminución en la inflamación, en el color rojo del tejido y costra a nivel de la semimucosa labial, lo que indica buen proceso de cicatrización (Figura 5B), después de 4 meses no presentó recidiva.

\section{DISCUSIÓN}

El GTB es una lesión reactiva común que puede aparecer a cualquier edad, se presenta con mayor frecuencia en mujeres, especialmente durante el embarazo, se observa en respuesta a una irritación o traumatis- mo, cálculos dentales, placa bacteriana y restauraciones [7], con respecto a población infantil, Vivas y otros en el 2013 afirmaron que con menor frecuencia se puede presentar en edades tempranas y no tiene predilección por genero sexual [9].

Con relación a las características clínicas Chen y otros en el 2021, Andreadis y otros en el 2019 reportaron que inicialmente puede presentarse como una pápula y con el pasar de los días se convierte en una lesión tumoral, de crecimiento exofítico, la base puede ser sésil o pedunculada, con una superficie irregular $y$ frecuentemente acompañada de sangrado $[10,11]$; aspectos clínicos que se presentaron en los dos casos reportados, con lesiones de aspecto tumoral, color roso, base pediculada, superficie irregular y sangrado espontáneo.

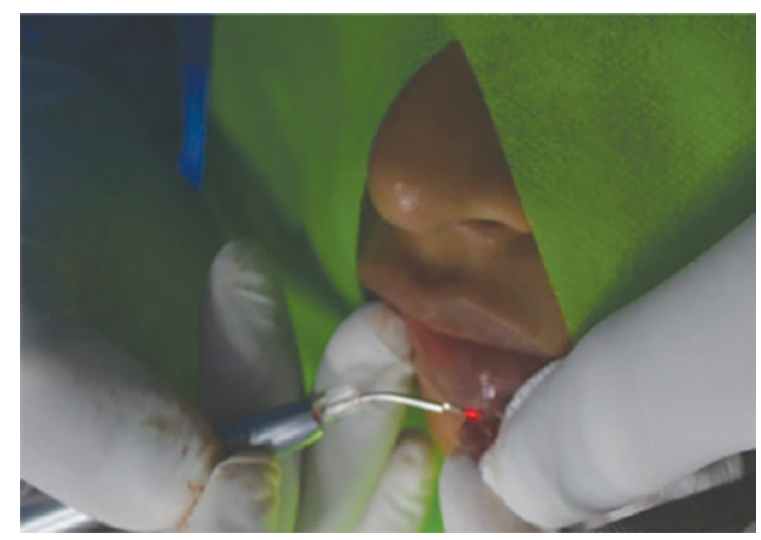

Figura 2. Remoción quirúrgica de la lesión con láser diodo. Fuente: Autores.

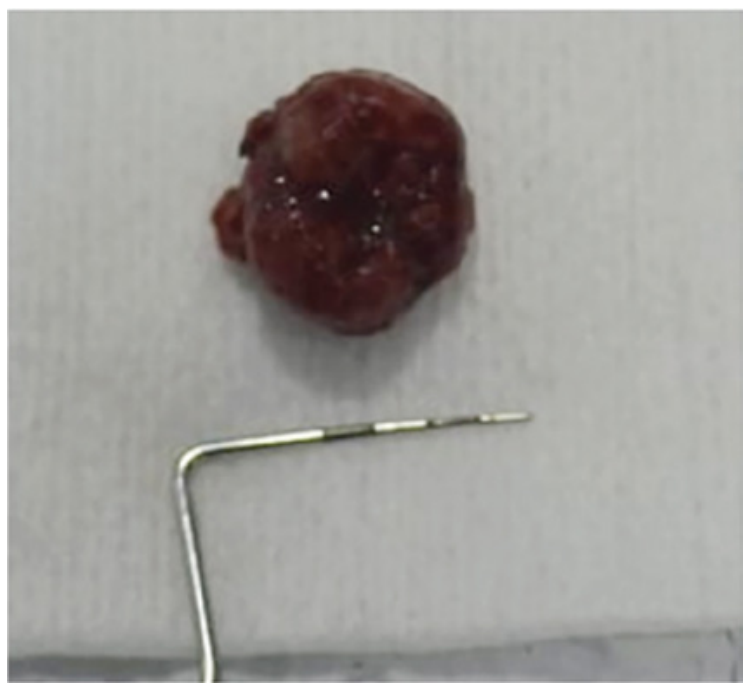

Figura 3. Muestra patológica. Fuente: Autores. 


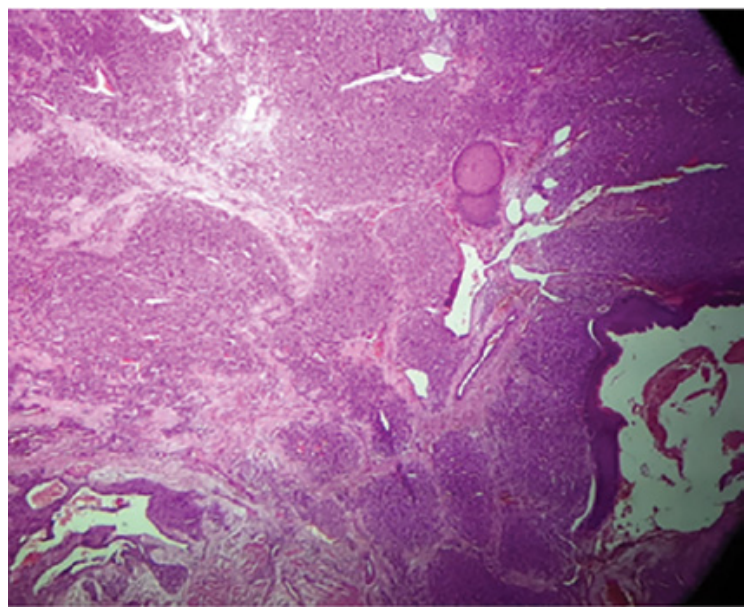

Figura 4. Corte histológico con epitelio escamoso hiperqueratósico, infiltrado inflamatorio con predominio de neutrófilos y abundantes vasos capilares. Fuente: Autores.
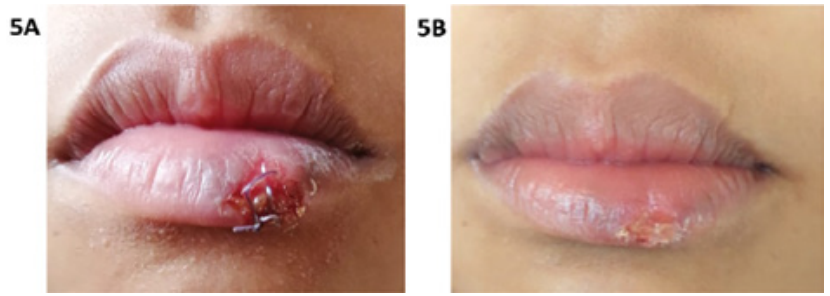

Figura 5A. Control clínico a los 8 días. Figura 5B. Control clínico a los 15 días.

El tratamiento quirúrgico consiente en niños es complicado, por todos los elementos cortopunzantes que se utilizan, como agujas, bisturí, tijeras, los cuales generan ansiedad, temor $\mathrm{y}$ angustia en el paciente, $\mathrm{Pi}$ sano y otros en el 2021 extirparon quirúrgicamente con láser de diodo el GTB en un paciente pediátrico, reportaron que el láser se percibe como una herramienta menos invasiva que el bisturí, con reducción del número de instrumentos quirúrgicos y es mejor aceptado por los niños [12]; concordando con el actual caso donde el niño toleró adecuadamente el tratamiento quirúrgico, a pesar de mostrarse temeroso en la consulta previa a la cirugía.

Espitia y otros en el 2018 reportaron el caso de un paciente con GTB en el que se aplicó láser diodo como tratamiento, en los resultados destacaron que el láser de diodo se mostró como óptima terapéutica, con diversas ventajas como fácil aplicación, mejor coagulación y buena respuesta biológica en la cicatrización [8]. Pisano y otros en el 2021 extirparon quirúrgicamente el GTB localizado en labio inferior de un paciente pediátrico, con láser de diodo, destacaron que la lesión presentó tendencia al sangrado, entre los resultados quirúrgicos de la técnica con láser afirmaron que la hemostasia fue efectiva, con mejor control del sangrado y buena cicatrización [12]; coincidiendo con el presente reporte de paciente con GTB sangrante, localizado en mucosa labial inferior, el cual fue tratado quirúrgicamente aplicando láser diodo de $940 \mathrm{~nm}$, con resultados positivos con relación al control del sangrado, el tiempo quirúrgico fue menor y con buena cicatrización. Los resultados, respecto al manejo del sangrado se deben a que la energía láser interactúa térmicamente con el colágeno de los vasos sanguíneos minimizando el sangrado [13].

\section{CONCLUSIÓN}

Según los resultados del presente caso clínico, el tratamiento del GTB con láser de diodo de $940 \mathrm{~nm}$ podría contemplarse como una alternativa terapéutica eficaz, por el modo de corte en el tejido, el control del sangrado, facilidad de uso y después de 4 meses no presenta recidiva.

\section{REFERENCIAS}

[1] Gadea C, Cartagena A, Cáceres-La Torre A. Diagnóstico y tratamiento del granuloma piógeno oral: una serie de casos. Rev Odontol Mex. 2017;21(4):244-52. DOI: 10.1016/j.rodmex.2018.01.006

[2] Asnaashari M, Mehdipour M, MoradiAbbasabadi F, Azari-Marhabi S. Expedited removal of pyogenic granuloma by diode laser in a pediatric patient. J Lasers Med Sci. 2015 Winter;6(1):40-4.

[3] Rucha D, Yusuf C, Abdul M, Rohan T. Oral telangiectatic granuloma. Universal Research Journal of Dentistry. 2016;6(2):182.

[4] Parajuli R, Maharjan S Unusual presentation of oral pyogenic granulomas: a review of two cases. Clinical Case Reports. 2018;6(4):690-693. DOI: 10.1002/ccr3.1435

[5] Patrón-Lambraño S, Martínez-Benavides M, Campo-Pacheco L, Ahumada-Montes A, Harris-Ricardo J. Granuloma telangiectásico en cavidad oral: informe de dos casos. Rev Fac Odontol Univ Cuenca. 2020;12:44-47.

[6] Rathore A, Jadhav T, Kulloli A, Singh A. Oral telangiectatic granuloma with an intrabony defect. J Indian Soc Periodontol. 2015;19(6):705-708.

[7] Tovio-Martínez E, del Valle-Urbano S, Díaz-Caballero A. Granuloma telangiectásico bucal. Rev Cubana Estomatol. 2018;55(1):82-90. 
[8] Espitia-Nieto S, Rebolledo-Cobos M, Cabrera-Meneses S, Carrascal-Restrepo L, Cuello-Noriega L. Láser de diodo: Tratamiento efectivo de inusual granuloma gravídico múltiple recidivante. Salud Uninorte. 2018;34(1):228-233.

[9] Vivas P, Da Silva De Carballo L, López-Labady J. Granuloma piogénico en la edad pediátrica - Reporte de un caso. Acta Odont Venez. 2013;51(2):1-6.

[10] Chen TL, Wang XM, Zhang XH, Chen J, Liu J. Therapeutic effects of diode laser on vascular epulis in esthetic area. J Indian Soc Periodontol. 2021;25(1):75-77. DOI: 10.4103/jisp.jisp_591_19

[11] Andreadis D, Lazaridi I, Anagnostou E, Poulopoulos A, Panta P, Patil S. Diode laser assisted excision of a gingival pyogenic granuloma: A case report. Clin Pract. 2019;9(3):1179. DOI: 10.4081/cp.2019.1179
[12] Pisano M, Sammartino P, Di Vittorio L, Iandolo A, Caggiano M, Roghi M, Bizzoca M, Lo Muzio L. Use of Diode Laser for Surgical Removal of Pyogenic Granuloma of the lower Lip in a Pediatric Patient: A Case Report. Am J Case Rep. 2021;22: e929690. DOI: 10.12659/ AJCR.929690

[13] Luke AM, Mathew S, Altawash MM, Madan BM. Lasers: A Review With Their Applications in Oral Medicine. J Lasers Med Sci. 2019;10(4):324-329. DOI: $10.15171 /$ jlms.2019.52

\section{Correo de autores}

María Burgos Anaya: mburgosa11@curnvirtual.edu.co

Zuly Garaviz Álvarez: zgaraviza17@curnvirtual.edu.co

Camila Giraldo Quintana: cgiraldoq17@curnvirtual.edu.co

José Llamas García: jose.llamas@curnvirtual.edu.co

David Rico Velasco: david.rico@curnvirtual.edu.co

Jonathan Harris Ricardo: Jonathan.harris@curnvirtual.edu.co 